\section{MATGEN: An interactive BASIC program for similarities data matrix generation}

\author{
DOUGLAS A. WELDON \\ Research Services Institute, Bay Shore, New York \\ and \\ RONALD BUCHTER \\ Lindenhurst Public Schools, Lindenhurst, New York
}

Multidimensional scaling (MDS) requires data indicating proximities among stimuli as input. A commonly used procedure for obtaining proximities data for large stimulus sets is stimulus sorting. This procedure requires subjects to place stimuli into mutually exclusive categories so that the stimuli within a category are judged to be more similar to each other than to stimuli in other categories.

Although this sorting task is very easy for subjects, it requires the rather tedious transformation of the grouped stimuli into a form acceptable as program input. The raw data must be transcribed into subject matrices before being submitted to MDS for analysis.

Description. MATGEN is a data matrix generation program designed to process free-sorted similarities data in preparation for submission to MDS. The program converts sorted data into matrices that are identified by subject numbers. The dimensions of each matrix are equal to the number of stimuli sorted. Each cell of a matrix contains either a " 1 ," indicating a common grouping of the stimuli that intersect at that cell, or a " 0 ," indicating that the intersecting stimuli were placed in different groups. A running tally of data sets is provided in a cumulative matrix that is updated as each subject's datum is

The first author's mailing address is: Research Services Institute, 967 Gardiner Drive, Bay Shore, NY 11706. The second author is with Lindenhurst Public Schools, Lindenhurst, NY 11757. processed, and a proportions matrix is generated upon completion of processing for all subjects. The program is dimensioned to handle up to 50 variables, but can be modified to handle larger data sets by changing array dimensioning statements.

Input. The stimuli are numbered from 1 to $\mathrm{n}$, where $\mathrm{n}$ is the total number of stimuli sorted. Input is facilitated by placing stimulus numbers in data statements. A " -1 " is placed at the end of each group of stimuli for a given subject, and a " 0 " is placed at the end of each subject's string of stimulus groups. Neither the order in which the stimuli appear in a group nor the order in which the groups appear in a string is of importance. After all stimulus groups have been placed in data statements, the program requests information concerning how many stimuli will be processed and whether or not individual and cumulative matrices are to be printed.

Output. If all printing options are selected, one $n \times n$ similarities matrix is printed for each subject, followed by one $n \times n$ cumulative matrix indicating total cell entries updated for each subject who has been processed. After all data have been processed, a proportions matrix that indicates similarities averaged over all subjects is printed. In addition, a warning is printed if any one of three errors has been made in entering data statements: (1) the same stimulus has been entered in two different groups; (2) a stimulus has been omitted for a subject; (3) the number of variables to be processed does not match the number indicated by the user.

Language. The program is written in BASIC, but uses no special matrix statements. Nested FOR-NEXT loops are used to process each matrix. This should enable the program to be implemented on a wide variety of systems, including both micro- and minicomputers.

Availability. A program listing can be obtained without charge from the first author.

(Revision accepted for publication December 7, 1984.) 